Document downloaded from:

http://hdl.handle.net/10251/79595

This paper must be cited as:

Herrero Debón, A.; Francisco J. Ramírez; Thome, N. (2014). An algorithm to study the nonnegativity, regularity and stability via state-feedbacks of singular systems of arbitrary index. Linear and Multilinear Algebra. 1-11. doi:10.1080/03081087.2014.904559.

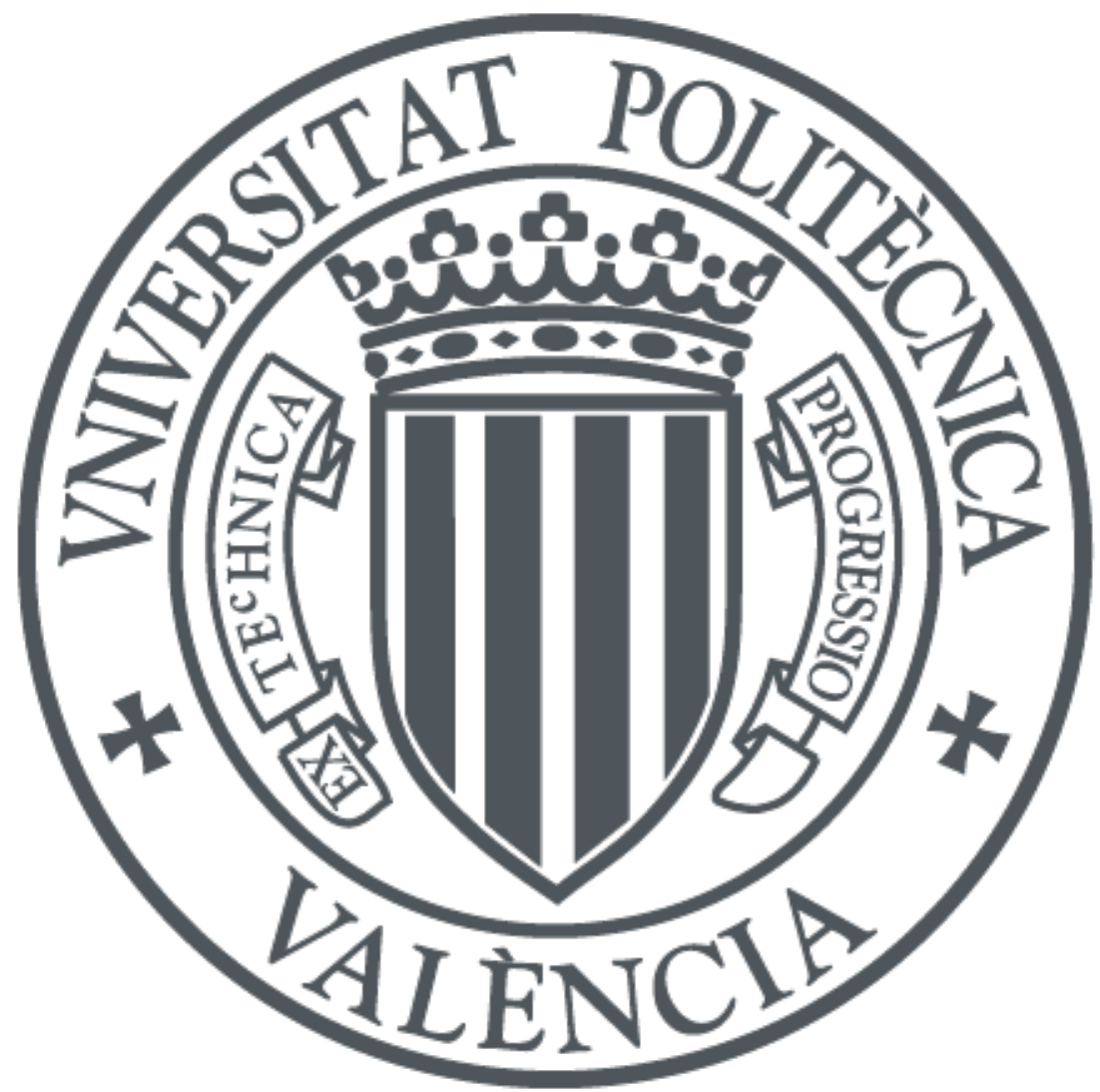

The final publication is available at

http://dx.doi.org/10.1080/03081087.2014.904559

Copyright Taylor \& Francis

Additional Information 


\title{
An algorithm to study the nonnegativity, regularity, and stability via state-feedbacks of singular systems of arbitrary index
}

\author{
A. Herrero* F. J. Ramírez ${ }^{\dagger} \quad$ Néstor Thome $^{\ddagger}$
}

July 2, 2015

\begin{abstract}
This paper deals with singular systems of index $k \geq 1$. Our main goal is to find a state-feedback such that the closed-loop system satisfies the regularity condition and it is nonnegative and stable. In order to do that, the core-nilpotent decomposition of a square matrix is applied to the singular matrix of the system. Moreover, if the Drazin projector of this matrix is nonnegative then the previous decomposition allows us to write the core-part of the matrix in a specific block form. In addition, an algorithm to study this kind of systems via a state-feedback is designed.
\end{abstract}

Keywords: Control system; nonnegativity; state-feedback; stability AMS Classification: 15A09 ; 93C05

*Instituto Universitario de Matemática Multidisciplinar, Universitat Politècnica de València, E-mail: aherrero@mat.upv.es.

†Instituto Tecnológico de Santo Domingo, Dominican Republic, E-mail: framiresj@hotmail.com.

${ }_{\ddagger}^{\ddagger}$ Instituto Universitario de Matemática Multidisciplinar, Universitat Politècnica de València, 46022, València, Spain. E-mail: njthome@mat.upv.es. This author was partially supported by Ministry of Education of Spain (grant DGI MTM2010-18228) and by Universidad Nacional de La Pampa, Argentina, Facultad de Ingeniería (grant Resol. No 049/11). 


\section{Introduction}

Nonnegative control systems appear in a wide range of areas such as: engineering, economical problems, electrical, mechanical, and chemical processes $[5,24,30]$. The property of nonnegativity plays an important role in these applications. These systems are dynamical systems whose state variables are nonnegative at all times. In $[13,18,25]$, the positive (nonnegative) singular systems have been widely developed.

Regularization of singular systems via state-feedbacks has been studied by different authors $[10,23,27]$. In general, those studies are based on the Weierstrass-Kronecker decomposition of the system, which uses two matrices $P$ and $Q$ that may change the information the original matrices. In this paper, we will use a different approach based on rearranging the information involved in the original matrices.

Stability of linear systems has been recently studied for autonomous descriptor systems in [20,26], for positive descriptor systems in [19,31], and for general linear systems in [2]. In both papers [2] and [26], special attention has been paid to nonnegativity of systems. Furthermore, some real problems have been treated in [9]. The design techniques proposed in this last case are based on the theory of externally nonnegative systems. Numerical aspects of singularity in systems can be found in [20, 28, 34, 38, 39] and those related to compute Drazin inverses in $[21,29,33]$.

In some applications the evolution of the system is represented in the state-space form and it is usual to look for feedbacks which transform it into a new system with specific properties, such as stability, symmetry, etc. In this paper we are interested on finding feedbacks that transform the original system into a new system satisfying the regularity condition, nonnegativity, and stability.

For a given matrix $A \in \mathbb{R}^{n \times n}$, a matrix $X \in \mathbb{R}^{n \times n}$ is called its Drazin inverse if the properties $X A X=X, A X=X A$, and $A^{q+1} X=A^{q}$ hold, where $q=\operatorname{ind}(A)$ is the index of $A$, that is, the smallest nonnegative integer such that $\operatorname{rank}\left(A^{q+1}\right)=\operatorname{rank}\left(A^{q}\right)$. The matrix $X$ always exists, it is unique

and denoted by $A^{D}[1,6,32]$. This generalized inverse matrix has been used to characterize the properties of nonnegative singular systems $[3,7]$; in both papers the authors used the whole coefficient matrices. An important subclass corresponds to the $q=1$ case, where the generalized inverse is called group inverse of $A$ and denoted by $A^{\#}$. We call Drazin projector of a square matrix $A$ to the matrix $A A^{D}$. Moreover, the Moore-Penrose inverse of a 
matrix $A \in \mathbb{R}^{m \times n}$ is the unique matrix $X \in \mathbb{R}^{n \times m}$ that satisfies $A X A=A$, $X A X=X,(A X)^{T}=A X$, and $(X A)^{T}=X A$. This generalized inverse always exists, it is unique and denoted by $X=A^{\dagger}[1,6]$.

Some extensions of results related to Drazin inverses on operator theory and Banach algebras have been presented, for example, in [11, 22]. Also, the Drazin inverse perturbation theory has been studied from different points of view. For instance, in [8, 37] algebraic approaches has been given while a setting in systems theory can be found in $[4,35,36]$.

We will stand $A \geq O$ for a matrix $A$ with nonnegative entries. The symbols $R(A)$ and $N(A)$ will denote the range and the null space of the matrix $A$ and $\sigma(A)$ the spectrum of a square matrix $A$. We will use the set $\sigma(E, A)=\{\lambda \in \mathbb{C}: \operatorname{det}(A-\lambda E)=0\}$ where $E, A \in \mathbb{R}^{n \times n}$. As usual, the open ball with center $a \in \mathbb{C}$ and radius $r>0$ is defined by $\mathcal{B}(a, r)=\{z \in$ $\mathbb{C}:|z-a|<r\}$.

This paper is organized as follows. In Section 2 we will state the problem of nonnegativity for singular systems via state-feedbacks. Moreover, some preliminary results on matrices with nonnegative Drazin projector are also included. In Section 3, necessary and sufficient conditions for the existence of feedbacks such that the closed-loop system is nonnegative, regular, and stable are obtained. The solution of this closed-loop system is also constructed in this section. Finally, in Section 4, we give an algorithm to construct the aforementioned feedback and examples that illustrate the results.

\section{Statement of the problem}

In this paper we consider discrete-time singular control systems like:

$$
\left\{\begin{aligned}
E x(k+1) & =A x(k)+B u(k) \\
y(k) & =C x(k)
\end{aligned}\right.
$$

where $E, A \in \mathbb{R}^{n \times n}, B \in \mathbb{R}^{n \times m}, C \in \mathbb{R}^{p \times n}, x(k) \in \mathbb{R}^{n \times 1}, u(k) \in \mathbb{R}^{m \times 1}$ and $y(k) \in \mathbb{R}^{p \times 1}$ with $\operatorname{rank}(E)=r<n$. In general, this system is denoted by $(E, A, B, C)$ or by $(E, A, C)$ when $B=O$. A wide analysis of singular systems can be found in [12] where structural properties, pole assignment and regularization of these systems are studied.

The system $(E, A, B, C)$ is said to satisfy the regularity condition if there exists a scalar $\alpha$ such that $\operatorname{det}(\alpha E+A) \neq 0$. In this case, the system (1) has solution. For further details, we refer the reader, for example, to [17]. 
On the other hand, it is well known that a matrix $E \in \mathbb{R}^{n \times n}$ of positive rank and positive index can be written as $E=B_{E}+N_{E}$ where

$$
B_{E}=S\left[\begin{array}{ll}
C & O \\
O & O
\end{array}\right] S^{-1} \quad \text { and } \quad N_{E}=S\left[\begin{array}{cc}
O & O \\
O & N
\end{array}\right] S^{-1}
$$

with $S, C$ nonsingular matrices and $N$ nilpotent. Note that $B_{E}$ has index 1 , $N_{E}$ is also nilpotent and $B_{E} N_{E}=N_{E} B_{E}=O$. Expression (2) is called the core-nilpotent decomposition of the matrix $E$ [6]. Note that the nilpotent part disappears when the index of $E$ equals 1.

Throughout the paper, we consider systems $(E, A, B, C)$ which may not satisfy the regularity condition and its matrix $E$ has nonnegative Drazin projector. In [16], under these conditions, it has been stated that the matrix $E$ can be written by means of the core-nilpotent decomposition as $E=$ $B_{E}+N_{E}$ where $N_{E}$ is a nilpotent matrix and

$$
P B_{E} P^{T}=\left[\begin{array}{c}
I \\
O \\
S
\end{array}\right] X T Y\left[\begin{array}{lll}
I & M & O
\end{array}\right]
$$

where $P$ is a permutation matrix, $P^{T}$ its transpose, $T \in \mathbb{R}^{r \times r}$ is a nonsingular matrix, $X=\operatorname{diag}\left(x_{1}, x_{2}, \ldots, x_{r}\right), Y=\operatorname{diag}\left(y_{1}^{T}, y_{2}^{T}, \ldots, y_{r}^{T}\right), x_{i}$ and $y_{i}$ are unit positive vectors such that $Y X=I$, and $M, S$ are nonnegative matrices of sizes $q \times s$ and $t \times q$, respectively. Notice that expression (3) has a block structure conformable to $n \times n=(q+s+t) \times(q+s+t)$. We also remark that the decomposition (3) appeared firstly in [14]. Later, the authors studied decomposition (3) and applications in [16].

On the other hand, expression (3) allows us to define the function

$$
\Psi_{M, S}: \mathbb{R}^{q \times q} \rightarrow \mathbb{R}^{n \times n}
$$

as

$$
\Psi_{M, S}(K)=\left[\begin{array}{c}
I \\
O \\
S
\end{array}\right] K\left[\begin{array}{lll}
I & M & O
\end{array}\right]
$$

where the matrices $M, S$, and $K$ are conformable for multiplication. Clearly, $P B_{E} P^{T}=\Psi_{M, S}(X T Y)$. Using block products of matrices, it is possible to deduce the following result.

Lemma 1. The function $\Psi_{M, S}$ previously defined satisfies the following properties: 
(a) $\Psi_{M, S}\left(K_{1}+K_{2}\right)=\Psi_{M, S}\left(K_{1}\right)+\Psi_{M, S}\left(K_{2}\right)$, for every $K_{1}, K_{2} \in \mathbb{R}^{q \times q}$.

(b) $\Psi_{M, S}(\mu K)=\mu \Psi_{M, S}(K)$, for every $\mu \in \mathbb{R}$ and $K \in \mathbb{R}^{q \times q}$.

(c) $\Psi_{M, S}\left(K_{1} K_{2} \ldots K_{l}\right)=\Psi_{M, S}\left(K_{1}\right) \Psi_{M, S}\left(K_{2}\right) \ldots \Psi_{M, S}\left(K_{l}\right)$, for every $K_{1}, \ldots, K_{l} \in$ $\mathbb{R}^{q \times q}$. In particular, $\Psi_{M, S}\left(K^{s}\right)=\left(\Psi_{M, S}(K)\right)^{s}$, for every $K \in \mathbb{R}^{q \times q}$ and $s \in \mathbb{N}$.

Now, using the permutation matrix $P$ appearing in (3), the singular system $(E, A, B, C)$ can be transformed by means of the change of state variable $z(k)=\operatorname{Px}(k)$ into the equivalent system $(\widetilde{E}, \widetilde{A}, \widetilde{B}, \widetilde{C})$ where

$$
\widetilde{E}=P E P^{T}, \quad \widetilde{A}=P A P^{T}, \quad \widetilde{B}=P B, \quad \widetilde{C}=C P^{T} .
$$

Clearly, some properties of the matrix $E$ are inherited by the matrix $\widetilde{E}$. For example, $E$ and $\widetilde{E}$ have the same index and the Drazin projector of $\widetilde{E}$ is also nonnegative. Using Lemma 1 and the core-nilpotent decomposition of $E$, the following result gives some expressions related to $\widetilde{E}$.

Lemma 2. The following properties hold:

(a) $\widetilde{E}=\Psi_{M, S}(X T Y)+P N_{E} P^{T}$ where $\Psi_{M, S}(X T Y) P N_{E}=N_{E} P^{T} \Psi_{M, S}(X T Y)=$ $O$.

(b) $\widetilde{E}^{D}=\Psi_{M, S}\left(X T^{-1} Y\right)$ where $P N_{E} P^{T} \widetilde{E}^{D}=\widetilde{E}^{D} P N_{E} P^{T}=O$.

(c) $\widetilde{E} \widetilde{E}^{D}=\Psi_{M, S}(X Y) \geq O$.

Notice that properties (a) and (b) in Lemma 2 can be deduced from the facts $B_{E} N_{E}=N_{E} B_{E}=O$ and $\Psi_{M, S}\left(X T^{-1} Y\right)=P B_{E}^{\#} P^{T}$. Moreover, we remark that property (a) becomes $\widetilde{E}=\Psi_{M, S}(X T Y)$ when the index of $E$ equals 1 .

For the system (4), we are going to construct a state-feedback $u(k)=$ $F z(k)$ such that the closed-loop system $(\widetilde{E}, \widetilde{A}+\widetilde{B} F, \widetilde{C})$ satisfies the regularity condition, is nonnegative and stable. We recall that a system $(E, A, B, C)$ is called nonnegative if $x(0) \geq 0$ and $u(k) \geq 0$ for all $k$ imply $x(k) \geq 0$ and $y(k) \geq 0$ for all $k$. Furthermore, a system $(E, A, B, C)$ is said to be stable if $\sigma(E, A) \subset \mathcal{B}(0,1)$. 


\section{Constructing the feedback}

As we have seen previously, the original system $(E, A, B, C)$ given by (1) can be transformed into the equivalent system $(\widetilde{E}, \widetilde{A}, \widetilde{B}, \widetilde{C})$ by means of the change of variable $z(k)=P x(k)$ as indicated in (4). In this way, the feedback $u(k)=F z(k)$ gives the closed-loop system $(\widetilde{E}, \widetilde{A}+\widetilde{B} F, \widetilde{C})$.

In order to achieve the regularity condition we can search for an adequate matrix $F$ such that

$$
\widetilde{A}+\widetilde{B} F=I-\beta \widetilde{E}
$$

holds.

Proposition 3. For a given $\beta \in \mathbb{R}$, there exists a feedback $F \in \mathbb{R}^{m \times n}$ satisfying equation (5) if and only if $R(I-\beta E-A) \subseteq R(B)$. In this case, the most general form for $F$ is

$$
F=\widetilde{B}^{\dagger}(I-\beta \widetilde{E}-\widetilde{A})+\left(I-\widetilde{B}^{\dagger} \widetilde{B}\right) Z,
$$

where $Z$ is an arbitrary matrix of adequate size.

Proof. For a given $\beta \in \mathbb{R}$, the matrix equation $\widetilde{B} F=I-\beta \widetilde{E}-\widetilde{A}$ has solution if and only if $\widetilde{B} \widetilde{B}^{\dagger}(I-\beta \widetilde{E}-\widetilde{A})=I-\beta \widetilde{E}-\widetilde{A}$ (see [1]), that is equivalent to

$$
\left(I-\widetilde{B} \widetilde{B}^{\dagger}\right)(I-\beta \widetilde{E}-\widetilde{A})=O .
$$

By using properties of the Moore-Penrose inverse, the last equation is equivalent to $R(I-\beta \widetilde{E}-\widetilde{A}) \subseteq N\left(I-\widetilde{B} \widetilde{B}^{\dagger}\right)=R\left(\widetilde{B} \widetilde{B}^{\dagger}\right)=R(\widetilde{B})$ since $\widetilde{B} \widetilde{B}^{\dagger}$ is a projector. This means that $R\left(I-\beta P E P^{T}-P A P^{T}\right) \subseteq R(P B)$, that is $R(I-\beta E-A) \subseteq R(B)$, where the information has been expressed in terms of the original matrices $E, A$ and $B$. Hence, by Theorem 1 in [1, pp. 52], we have that $F$ has the form given in (6).

The arbitrariness of the matrix $Z$ in expression (6) produces all the possible feedbacks for the selected value of $\beta$ in (5). If we need to compute only one feedback we can clearly take $Z=O$.

With regard to the nonnegativity of the closed-loop system, we have to study the conditions: $\widetilde{E} \widetilde{E}^{D} \geq O, \widetilde{E}^{D}(\widetilde{A}+\widetilde{B} F) \geq O$ and $\widetilde{C} \widetilde{E} \widetilde{E}^{D} \geq O[15$, Theorem 2.1].

The first condition is satisfied by Lemma 2 and the analysis of the other two conditions lead to the following result. 
Theorem 4. Let $(E, A, B, C)$ be the system given by (1). The closed-loop system $(\widetilde{E}, \widetilde{A}+\widetilde{B} F, \widetilde{C})$ constructed with the feedback $u(k)=F z(k)$ and the matrices given in (4) is regular and nonnegative if there exists a scalar $\beta$ such that $\widetilde{A}+\widetilde{B} F=I-\beta \widetilde{E}$ and the following conditions hold:

a) $T^{-1}-\beta I \geq O$,

b) $\left(C_{1}+C_{3} S\right) X \geq O$,

where $\widetilde{C}=\left[\begin{array}{lll}C_{1} & C_{2} & C_{3}\end{array}\right]$ according to the blocks of $\Psi_{M, S}(X Y)$ with $M, S, X, Y$ and $T$ as in (3).

Proof. Assume that there exists a scalar $\beta$ such that $\widetilde{A}+\widetilde{B} F=I-\beta \widetilde{E}$. We have to analyze the conditions $\widetilde{E}^{D}(\widetilde{A}+\widetilde{B} F) \geq O$ and $\widetilde{C} \widetilde{E} \widetilde{E}^{D} \geq O$.

Since $\widetilde{E}^{D}(\widetilde{A}+\widetilde{B} F)=\widetilde{E}^{D}(I-\beta \widetilde{E})$ and $\widetilde{E}^{D}=\Psi_{M, S}\left(X T^{-1} Y\right)$ by Lemma 2 , we have that the first condition is equivalent to

$$
\Psi_{M, S}\left(X T^{-1} Y\right)\left[I-\beta \Psi_{M, S}(X T Y)\right] \geq O .
$$

Thus, Lemma 1 allows us to write inequality (7) as

$$
\Psi_{M, S}\left(X\left(T^{-1}-\beta I\right) Y\right) \geq O,
$$

where we have used that $Y X=I$. This last inequality implies that the block $(1,1)$ of $\Psi_{M, S}\left(X\left(T^{-1}-\beta I\right) Y\right)$ has to be nonnegative, that is $X\left(T^{-1}-\beta I\right) Y \geq$ $O$. Since $X, Y \geq O$ and $Y X=I$, we get $T^{-1}-\beta I \geq O$. Notice that the only block that gives information on the nonnegativity is the block $(1,1)$ due to $M$ and $S$ are nonnegative matrices.

Related to the condition $\widetilde{C} \widetilde{E} \widetilde{E}^{D} \geq O$ to be analyzed to assure the nonnegativity of the system, by Lemma 2 we have that it is equivalent to

$$
\widetilde{C} \Psi_{M, S}(X Y) \geq O .
$$

Partitioning the matrix $\widetilde{C}$ according to the sizes of the blocks of $\Psi_{M, S}(X Y)$ as

$$
\widetilde{C}=C P^{T}=\left[\begin{array}{lll}
C_{1} & C_{2} & C_{3}
\end{array}\right]
$$

we get

$$
\left[\begin{array}{lll}
C_{1} & C_{2} & C_{3}
\end{array}\right]\left[\begin{array}{c}
I \\
O \\
S
\end{array}\right] X Y\left[\begin{array}{lll}
I & M & O
\end{array}\right] \geq O
$$


Again, the block $(1,1)$ in the last matrix contains the main information to assure the nonnegativity of the entire matrix. This condition becomes $\left(C_{1}+C_{3} S\right) X \geq O$ taking into account that $X \geq O$ and $Y X=I$.

Next, we analyze the stability of the closed-loop system considering that the system is regular. The following result gives sufficient conditions for the stability of the system.

Theorem 5. Let $(E, A, B, C)$ be the system given by (1). The closed-loop system $(\widetilde{E}, \widetilde{A}+\widetilde{B} F, \widetilde{C})$ constructed with the feedback $u(k)=F z(k)$ and the matrices given in (4) is regular and stable if there exists a scalar $\beta \in \mathcal{B}(\gamma, 1)$ such that $\widetilde{A}+\widetilde{B} F=I-\beta \widetilde{E}$ for every $\gamma \in \sigma\left(T^{-1}\right)$ where $T$ is given in (3).

Proof. Since there exists a scalar $\beta$ such that $\widetilde{A}+\widetilde{B} F=I-\beta \widetilde{E}$, the regularity condition holds.

On the other hand, the closed-loop system is stable if and only if the set $\sigma(\widetilde{E}, \widetilde{A}+\widetilde{B} F) \subset \mathcal{B}(0,1)$. By definition, the set $\sigma(\widetilde{E}, \widetilde{A}+\widetilde{B} F)$ is given by the $\lambda$ 's such that

$$
0=\operatorname{det}(\lambda \widetilde{E}-I+\beta \widetilde{E})=\operatorname{det}(\gamma \widetilde{E}-I)=\gamma^{n} \operatorname{det}\left(\widetilde{E}-\frac{1}{\gamma} I\right)
$$

with $\gamma=\lambda+\beta \neq 0$, that is, $1 / \gamma \in \sigma(\widetilde{E})$. In order to get $\lambda \in \mathcal{B}(0,1)$, we must have $\beta \in \mathcal{B}(\gamma, 1)$ for every $\gamma$ such that $1 / \gamma \in \sigma(\widetilde{E})$.

By Lemma 2, we have $\sigma(\widetilde{E})=\sigma(X T Y) \cup\{0\}$. Moreover, $\sigma(X T Y)-\{0\}=$ $\sigma(T)$. In fact, if $\alpha \in \sigma(X T Y)-\{0\}$ then there exists $z \neq 0$ such that $X T Y z=\alpha z$. Premultiplying by $Y$ and using that $Y X=I$ we get that $T Y z=\alpha Y z$, so $\alpha \in \sigma(T)$ because $Y z \neq 0$. Similarly, if $\alpha \in \sigma(T)$ then there exists $z \neq 0$ such that $T z=\alpha z$ and, furthermore, $\alpha \neq 0$ because $T$ is nonsingular. Again, premultiplying by $X$ and using that $Y X=I$ we get that $X T Y X z=\alpha X z$, so $\alpha \in \sigma(X T Y)-\{0\}$ because $X z \neq 0$. Thus, $\sigma(X T Y)-\{0\}=\sigma(T)$.

Hence, the stability of the closed-loop system is guaranteed by the condition $\beta \in \mathcal{B}(\gamma, 1)$ for every $\gamma$ such that $1 / \gamma \in \sigma(T)$.

Up to now, we have analyzed conditions such that the closed-loop system $(\widetilde{E}, \widetilde{A}+\widetilde{B} F, \widetilde{C})$ satisfies the regularity, nonnegativity, and stability conditions. Under these assumptions, we present an explicit solution of the system where the nonnegativity of the states and outputs can be clearly checked. 
The solution of the closed-loop system is $y(k)=\widetilde{C} z(k)$ where $z(k)$ is given by [17]:

$$
z(k)=\left(\widetilde{E}^{D}(I-\beta \widetilde{E})\right)^{k} \widetilde{E}^{D} \widetilde{E} z(0)
$$

with $z(0) \in R\left(\left[\begin{array}{cc}\widetilde{E}^{D} \widetilde{E} \quad\left(I-\widetilde{E}^{D} \widetilde{E}\right)(I-\beta \widetilde{E})^{D}\end{array}\right]\right)$. This last set is the subspace of the initial admissible conditions of the system. As $\widetilde{E}^{D}$ and $I-\beta \widetilde{E}$ commute, the properties of the Drazin inverse allow us to write

$$
\begin{aligned}
z(k) & =\left((I-\beta \widetilde{E}) \widetilde{E}^{D}\right)^{k} z(0) \\
& =\left(\left(I-\beta \Psi_{M, S}(X T Y)-\beta P N_{E} P^{T}\right) \Psi_{M, S}\left(X T^{-1} Y\right)\right)^{k} z(0) \\
& =\Psi_{M, S}\left(X\left(T^{-1}-\beta I\right)^{k} Y\right) z(0)
\end{aligned}
$$

where we have used the definition of the function $\Psi_{M, S}$ and its properties given in Lemma 1. Hence, it is clear that the states $z(k)$ are nonnegative since $T^{-1}-\beta I \geq O$ and $z(0) \geq 0$.

Then, the outputs of the system are given by:

$$
\begin{aligned}
y(k) & =\left[\begin{array}{lll}
C_{1} & C_{2} & C_{3}
\end{array}\right] \Psi_{M, S}\left(X\left(T^{-1}-\beta I\right)^{k} Y\right) z(0) \\
& =\left(C_{1}+C_{3} S\right) X\left(T^{-1}-\beta I\right)^{k} Y\left[\begin{array}{lll}
I & M & O
\end{array}\right] z(0)
\end{aligned}
$$

where clearly $\left(C_{1}+C_{3} S\right) X \geq O$ and $T^{-1}-\beta I \geq O$ impliy $y(k) \geq 0$ for a nonnegative initial admissible condition.

\section{Algorithm and examples}

This section gives a procedure that systematizes the reasoning presented in Section 3. In order to do that, we provide an algorithm where the existence of a feedback that guarantees the regularity, nonnegativity, and stability of a system is analyzed. Moreover, the construction of such a feedback is also carried out.

\section{Algorithm:}

Inputs: A singular system $(E, A, B, C)$ that satisfies $E E^{D} \geq O$.

Outputs: Matrices $F$ such that the closed-loop system $(\widetilde{E}, \widetilde{A}+\widetilde{B} F, \widetilde{C})$ is regular, nonnegative, and stable, and the solution of this system. 
Step 1: Transform the original system $(E, A, B, C)$ into the equivalent system $(\widetilde{E}, \widetilde{A}, \widetilde{B}, \widetilde{C})$ given in (4).

Step 2: If $\left(C_{1}+C_{3} S\right) X \nsupseteq O$ then go to Step 10 .

Step 3: Compute $\sigma(T)$ and $\Lambda=\cap\{\mathcal{B}(\gamma, 1): 1 / \gamma \in \sigma(T)\}$.

Step 4: If $\Lambda \cap \mathbb{R}=\emptyset$ then go to Step 10 .

Step 5: Choose $\beta \in \Lambda \cap \mathbb{R}$. Note that in each step, we propose to try with a finite number of different values of $\beta$ else go to Step 10.

Step 6: If $T^{-1}-\beta I \nsupseteq O$ then go to Step 5 .

Step 7: Compute $\widetilde{B}^{\dagger}$.

Step 8: If $\left(I-\widetilde{B} \widetilde{B}^{\dagger}\right)(I-\beta \widetilde{E}-\widetilde{A}) \neq O$ then go to Step 5 or go to Step 10 .

Step 9: Construct $F=\widetilde{B}^{\dagger}(I-\beta \widetilde{E}-\widetilde{A})+\left(I-\widetilde{B}^{\dagger} \widetilde{B}\right) Z$ with $Z$ arbitrary. Go to Step 11.

Step 10: 'There exists no matrix $F$ such that the closed-loop system is nonnegative'. Go to End.

Step 11: The closed-loop system is regular, nonnegative and stable. The outputs of the system $(\widetilde{E}, \widetilde{A}+\widetilde{B} F, \widetilde{C})$ are given by

$$
y(k)=\left(C_{1}+C_{3} S\right) X\left(T^{-1}-\beta I\right)^{k} Y\left[\begin{array}{lll}
I & M & O
\end{array}\right] z(0) .
$$

\section{End}

We illustrate the obtained results with the following examples.

Example 6. Let $(E, A, B, C)$ be a singular system whose matrix $E=$ $\Psi_{M, S}(X T Y)+N_{E}$ is given by

$$
\Psi_{M, S}(X T Y)=\left[\begin{array}{ccccccccc}
3 & 0 & 0 & 0 & 3 & 6 & 0 & 0 & 0 \\
6 & 0 & 0 & 0 & 6 & 12 & 0 & 0 & 0 \\
-1 & 0 & 1 & 1 & -1 & -2 & 0 & 0 & 0 \\
-1 & 0 & 1 & 1 & -1 & -2 & 0 & 0 & 0 \\
0 & 0 & 0 & 0 & 0 & 0 & 0 & 0 & 0 \\
0 & 0 & 0 & 0 & 0 & 0 & 0 & 0 & 0 \\
8 & 0 & 7 & 7 & 8 & 16 & 0 & 0 & 0 \\
0 & 0 & 0 & 0 & 0 & 0 & 0 & 0 & 0 \\
42 & 0 & 6 & 6 & 42 & 84 & 0 & 0 & 0
\end{array}\right]=\left[\begin{array}{c}
I \\
O \\
S
\end{array}\right] X T Y\left[\begin{array}{lll}
I & M & O
\end{array}\right]
$$


with

$S=\left[\begin{array}{llll}1 & 2 & 3 & 4 \\ 0 & 0 & 0 & 0 \\ 0 & 8 & 2 & 4\end{array}\right], M=\left[\begin{array}{ll}1 & 2 \\ 3 & 4 \\ 0 & 0 \\ 0 & 0\end{array}\right], T=\left[\begin{array}{cc}3 & 0 \\ -1 & 2\end{array}\right], X=\left[\begin{array}{c|c}1 & 0 \\ 2 & 0 \\ \hline 0 & 1 \\ 0 & 1\end{array}\right], Y=\left[\begin{array}{cc|cc}1 & 0 & 0 & 0 \\ \hline 0 & 0 & 1 / 2 & 1 / 2\end{array}\right]$

and

$$
N_{E}=\left[\begin{array}{lllllllll}
0 & 1 & 0 & 0 & 0 & 0 & 0 & 0 & 0 \\
0 & 0 & 1 & 0 & 0 & 0 & 0 & 0 & 0 \\
0 & 0 & 0 & 1 & 0 & 0 & 0 & 0 & 0 \\
0 & 0 & 0 & 0 & 0 & 0 & 0 & 0 & 0 \\
0 & 0 & 0 & 0 & 0 & 0 & 0 & 0 & 0 \\
0 & 0 & 0 & 0 & 0 & 0 & 0 & 0 & 0 \\
0 & 0 & 0 & 0 & 0 & 0 & 0 & 0 & 0 \\
0 & 0 & 0 & 0 & 0 & 0 & 0 & 0 & 0 \\
0 & 0 & 0 & 0 & 0 & 0 & 0 & 0 & 0
\end{array}\right]
$$

Let

$$
A=\frac{1}{3}\left[\begin{array}{ccccccccc}
6 & 1 & 0 & 0 & 3 & 6 & 0 & 0 & 0 \\
12 & 3 & 18 & 12 & 12 & 24 & 0 & 0 & 0 \\
-1 & 0 & 4 & 2 & -1 & -2 & 0 & 0 & 0 \\
-1 & 0 & 1 & 4 & -1 & -2 & 0 & 0 & 0 \\
0 & 0 & 0 & 0 & 3 & 0 & 0 & 0 & 0 \\
0 & 0 & 0 & 0 & 0 & 3 & 0 & 0 & 0 \\
8 & 0 & 7 & 7 & 8 & 16 & 3 & 0 & 0 \\
0 & 0 & 0 & 0 & 0 & 0 & 0 & 3 & 0 \\
42 & 0 & 6 & 6 & 42 & 84 & 0 & 0 & 3
\end{array}\right], \quad B=\left[\begin{array}{c}
0 \\
-1 \\
0 \\
0 \\
0 \\
0 \\
0 \\
0 \\
0
\end{array}\right], \quad C=\left[\begin{array}{ccc}
C_{1} & C_{2} & C_{3}
\end{array}\right]
$$

with $C_{1}, C_{2}$, and $C_{3}$ arbitrary matrices satisfying $\left(C_{1}+C_{3} S\right) X \geq O$.

Note that Steps 1 and 2 of the algorithm hold. Now, we compute Step 3 obtaining

$$
\sigma(T)=\{2,3\} \quad \text { and } \quad \mathcal{B}(1 / 2,1) \cap \mathcal{B}(1 / 3,1) \cap \mathbb{R}=]-1 / 2,4 / 3[.
$$

Following with Steps 4,5 , and 6 , we have to choose $\beta \in]-1 / 2,4 / 3[$ such that

$$
T^{-1}-\beta I=\left[\begin{array}{cc}
1 / 3-\beta & 0 \\
1 / 6 & 1 / 2-\beta
\end{array}\right] \geq O \Leftrightarrow\left\{\begin{array}{c}
1 / 3-\beta \geq 0 \\
1 / 2-\beta \geq 0
\end{array}\right.
$$


So, for example, we can choose $\beta=-1 / 3$. Since $B^{\dagger}=B^{T}$ (Step 7), we have that $\left(I-B B^{\dagger}\right)(I-\beta E-A)=O$. Finally, Steps 9 and 11 provide the feedback and the solution of the closed-loop system which is regular, nonnegative, and stable.

We close this paper with a real example based on the Leontief model.

Example 7. A dynamic Leontief model of a multisector economy has the form $[12]$

$$
x(k)=L x(k)+C(x(k+1)-x(k))+D u(k)
$$

where $x(k)$ is the vector of output levels, $D u(k)$ is the vector of final demands (excluding investment), $L$ is the Leontief input-output matrix, and $C$ is the capital coefficient matrix. The matrices $L$ and $C$ are assumed to be known and time invariant. An essential property of these kind of models is the nonnegativity.

Considering three sector economy Leontief model with the following coefficient matrices:

$$
L=\left[\begin{array}{ccc}
0.5 & 0.6667 & 0.75 \\
0 & 0 & 0 \\
0.5 & 1.6667 & 1
\end{array}\right], \quad C=\left[\begin{array}{ccc}
0.3 & 0.4 & 0.45 \\
0 & 0 & 0 \\
0.6 & 0.8 & 0.9
\end{array}\right], \quad D=\left[\begin{array}{l}
0 \\
0 \\
1
\end{array}\right]
$$

we arrive at the singular system $C x(k+1)=(I-L+C) x(k)-D u(k)$, that is

$$
\left[\begin{array}{ccc}
0.3 & 0.4 & 0.45 \\
0 & 0 & 0 \\
0.6 & 0.8 & 0.9
\end{array}\right] x(k+1)=\left[\begin{array}{ccc}
0.8 & -0.2667 & -0.3 \\
0 & 1 & 0 \\
0.1 & -0.2667 & 0.9
\end{array}\right] x(k)-\left[\begin{array}{l}
0 \\
0 \\
1
\end{array}\right] u(k)
$$

which is not nonnegative. We apply the Algorithm to obtain a nonnegative, regular and stable closed-loop system. To do that, let

$$
\begin{gathered}
\widetilde{E}=P C P^{T}=\left[\begin{array}{c}
I \\
O
\end{array}\right] X T Y\left[\begin{array}{ll}
I & M
\end{array}\right]=\left[\begin{array}{ccc}
0.3 & 0.45 & 0.4 \\
0.6 & 0.9 & 0.8 \\
0 & 0 & 0
\end{array}\right], \\
\widetilde{A}=P(I-L+C) P^{T}=\left[\begin{array}{ccc}
0.8 & -0.3 & -0.2667 \\
0.1 & 0.9 & -0.2667 \\
0 & 0 & 1
\end{array}\right] \text { and } \widetilde{B}=P(-D)=\left[\begin{array}{c}
0 \\
-1 \\
0
\end{array}\right]
\end{gathered}
$$


where

$P=\left[\begin{array}{lll}1 & 0 & 0 \\ 0 & 0 & 1 \\ 0 & 1 & 0\end{array}\right], X=\left[\begin{array}{l}1 \\ 2\end{array}\right], Y=\left[\begin{array}{ll}0.25 & 0.375\end{array}\right], T=1.2, M=\left[\begin{array}{c}1.3333 \\ 0\end{array}\right]$

Since $\Lambda=\mathcal{B}\left(\frac{5}{6}, 1\right) \cap \mathbb{R} \neq \emptyset$, Steps 3 and 4 hold and we can choose $\beta=\frac{2}{3} \in \Lambda$ in Step 5. So, Step 6 holds and computing $\widetilde{B}^{\dagger}=\left[\begin{array}{lll}0 & -1 & 0\end{array}\right]$ we can check Step 8. Now, we can obtain $F$ directly from expression in Step 9 as desired.

\section{Acknowledgements}

This paper has been partially supported by Ministry of Education of Spain (grant DGI MTM2010-18228).

\section{References}

[1] A. Ben-Israel and T. Greville, Generalized Inverses: Theory and Applications, John Wiley \& Sons, $2^{\text {nd }}$ edition, New York, 2003.

[2] C. Briat, Robust stability and stabilization of uncertain linear positive systems via integral linear constraints: $L_{1}$-gain and $L_{\infty}$-gain characterization, International Journal of Robust and Nonlinear Control, DOI: 10.1002/rnc.2859, (2012).

[3] R. Bru, C. Coll and E. Sánchez, Structural properties of positive linear time-invariant difference-algebraic equations, Linear Algebra and Its Applications, 349 (2002), pp. 1-10.

[4] M. Busłowicz, Robust stability of positive discrete-time linear systems with multiple delays with linear unity rank uncertainty structure or nonnegative perturbation matrices, Bulletin of the Polish Academy of Sciences, Technical Sciences, 55, 1 (2007).

[5] S.L. Campbell, Singular systems of differential equations, Pitman, London, 1980.

[6] S.L. Campbell and C.D. Meyer, Jr., Generalized inverses of linear transformations, Dover, 1979. 
[7] B. Cantó, C. Coll and E. Sánchez, Positive solutions of a discrete-time descriptor system, International Journal of Systems Science 39, 1 (2008), pp. 81-88.

[8] N. Castro-González, J.J. Koliha and Y. Wei, Perturbation of the Drazin inverse for matrices with equal eigenprojections at zero, Linear Algebra and its Applications, 312, (2000) 181-189.

[9] L. Celentano, Tracking Controllers Design of References with Bounded Derivative, Applied Mathematical Sciences, 6, (2012), 95, pp. 4709-4728.

[10] C. Coll, A. Herrero, E. Sánchez and N. Thome, Output feedback stabilization for symmetric control systems, Journal of the Franklin Institute, 342 (2005), pp. 814-823.

[11] C. Deng, D. S. Cvetković-Ilić and Y. Wei, Some results on the generalized Drazin inverse of operator matrices, Linear and Multilinear Algebra, 58, 4 (2010), pp. 503-521.

[12] G.R. Duan, Analysis and design of descriptor linear systems. Advances in Mechanics and Mathematics, 23, Springer, New York, 2010.

[13] L. Farina and S. Rinaldi, Positive linear systems: Theory and applications. J. Wiley, New York, 2000.

[14] P. Flor, On groups of non-negative matrices, Compositio Math., 21, 4 (1969), pp. 376382.

[15] A. Herrero, A. Ramírez and N. Thome, An algorithm to check the nonnegativity of singular systems, Applied Mathematics and Computation 189 (2007), pp. 355-365.

[16] A. Herrero, F. Ramírez and N. Thome, Relationships between different sets involving group and Drazin projectors and nonnegativity, Linear Algebra and its Applications, 438 (2013), pp. 1688-1699.

[17] T. Kaczorek, Linear control systems, Vol. I and II, Wiley, 1992.

[18] T. Kaczorek, Positive $1 D$ and 2D Systems, Springer-Verlag, London, 2002. 
[19] T. Kaczorek, Stability of descriptor positive linear systems, COMPEL, 32, no.1 (2013), pp. 412-423.

[20] P. Kunkel and V. Mehrmann, Differential-algebraic equations. Analysis and numerical solution, EMS Textbooks in Mathematics. European Mathematical Society (EMS), Zürich, 2006.

[21] S. Miljković, M. Miladinović, P.S. Stanimirović and Y. Wei, Gradient methods for computing the Drazin-inverse solution, Journal of Computational Applied Mathematics, 253 (2013), pp. 255-263.

[22] D. Mosić and D.S. Djordjević, Representation for the generalized Drazin inverse of block matrices in Banach algebras, Applied Mathematics and Computation, 218 (2012), pp. 12001-12007.

[23] R. Mukundan and W. Dayawansa, Feedback control of singular systemsproportional and derivative feedback of the state, Internat. J. Systems Sci., 14 (1983), pp. 615-632.

[24] P.C. Müller, Linear mechanical descriptor systems: identification, analysis and design, in: Preprints of IFAC Conference on control of independent systems, Belfort, France, 1997, pp. 501-506.

[25] D. Noustos and M.J. Tsatsomeros, Reachability and holdability of nonnegative states, SIAM J. Matrix Analysis and Applications, 30, 2 (2008), pp. 700-712.

[26] M.A. Rami and D. Napp, Characterization and Stability of Autonomous Positive Descriptor Systems, IEEE Transactions on Automatic Control, 57, 10 (2012), pp. 2668-2673.

[27] A. Shayman and Z. Zhou, Feedback control and classification of generalized linear systems, IEEE Trans. Automat. Control, AC-32 (1987), pp. 483-494.

[28] X. Shi, Y. Wei and W. Zhang, Convergence of general nonstationary iterative methods for solving singular linear equations, SIAM J. Matrix Analisys and Applications, 32, 1 (2011), pp. 72-89.

[29] A. Sidi, DGMRES: a GMRES-type algorithm for Drazin-inverse solution of singular nonsymmetric linear systems, Linear Algebra and its Applications, 335 (2001), pp. 189-204. 
[30] M.S. Silva and T.P. de Lima, Looking for Nonnegative Solutions of a Dynamic Leontief Model, Linear Algebra and its Applications, 364 (2003), pp. 281-216.

[31] E. Virnik, Stability analysis of positive descriptor systems, Linear Algebra and its Applications, 429 (2008), pp. 2640-2659.

[32] G. Wang, Y. Wei and S. Qiao, Generalized inverses: Theory and Computations. Beijing: Science Press, 2004.

[33] Y. Wei, Index splitting for the Drazin inverse and the singular linear system, Applied Mathematics and Computation, 95, 2-3 (1998), pp. 115124 .

[34] Y. Wei and H. Wu, Convergence properties of Krylov subspace methods for singular linear systems with arbitrary index, Journal of Computational Applied Mathematics, 114, 2 (2000), pp. 305-318.

[35] Y. Wei and H. Wu, Additional results on index splittings for Drazin inverse solutions of singular linear systems, Electronic Journal of Linear Algebra, 8 (2001), pp. 83-93.

[36] S. Xu and J. Lam, Robust control and filtering of singular systems, Lecture Notes in Control and Information Sciences, 332, Springer-Verlag, Berlin, 2006.

[37] Q. Xu, C. Song and Y. Wei, The stable perturbation of the Drazin inverse of the square matrices, SIAM J. Matrix Anal. Appl., 31, no. 3 (2009), pp. $1507-1520$.

[38] N. Zhang, A note on preconditioned GMRES for solving singular linear systems. BIT, 50, 1 (2010), pp. 207-220.

[39] N. Zhang and Y. Wei, On the convergence of general stationary iterative methods for range-Hermitian singular linear systems. Numerical Linear Algebra and Applications, 17, 1 (2010), pp. 139-154. 